DOI 10.37882/2500-3682.2021.08.06

\title{
КОМПЛЕКСНЫЙ ПОДХОД К ВОССТАНОВЛЕНИЮ ВЫСШИХ ПСИХИЧЕСКИХ ФУНКЦИЙ У ПАЦИЕНТОВ ПОСЛЕ ОСТРОГО НАРУШЕНИЯ МОЗГОВОГО КРОВООБРАЩЕНИЯ
}

\section{A COMPREHENSIVE APPROACH TO THE RECOVERY OF HIGHER MENTAL FUNCTIONS IN PATIENTS AFTER ACUTE IMPAIRMENT OF CEREBRAL CIRCULATION \\ N. Zemtseva \\ L. Tishina}

Summary: Currently, the problem of restoring the functions of higher nervous activity in acute cerebrovascular accident due to an increase in the frequency of occurrence in the population, as well as the lack of safe and effective methods for correcting negative symptoms is becoming increasingly important. This article describes the main symptoms that arise with this anomaly, describes its complexity and heterogeneity. The main risk factors leading to the emergence of acute disorders of cerebral circulation are highlighted. The issues of diagnosis and recognition of negative symptoms are discussed in detail and a detailed description of the clinical case is provided using an individual approach to the convalescence of the functions of higher nervous activity. Separately, the importance of creating aspects of an integrated individual approach in the treatment of behavioral and cognitive disorders is emphasized, as well as methods, approaches and methods of treatment are proposed for correcting various clinical symptoms of this disease based on the analysis of the literature data.

Keywords: communicative function of speech, higher nervous activity, cognitive disorders, acute impairment of cerebral circulation, integrated approach.

\author{
Земцева Наталья Владимировна \\ Аспирант, Московский государственный \\ психолого-педагогический университет \\ nataliznv@mail.ru \\ тишина Людмила Александровна \\ К.п.н., дочент, Московский государственный \\ психолого-педагогический университет \\ tishinala@mgppu.ru
}

Аннотация: В настоящее время проблема восстановления функций высшей нервной деятельности при остром нарушении мозгового кровообращения в связи с ростом частоты встречаемости в популяции, а также отсутствием безопасных и эффективных способов коррекции негативной симптоматики приобретает всё большую актуальность. В рассматриваемой статье приводится описание основных симптомов, возникающих при этой аномалии, описывается её сложность и гетерогенность. Выделены основные факторы риска, приводящие к появлению острого нарушения мозгового кровообращения. Детально разобраны вопросы диагностики и распознавания негативной симптоматики и приводится подробное описание клинического случая с применением индивидуального подхода к реконвалесценции функций высшей нервной деятельности. Отдельно подчёркивается важность создания аспектов комплексного индивидуального подхода при лечении поведенческих и когнитивных нарушений, а также предлагаются способы, подходы и методы лечения, при коррекции разных клинических симптомов этого заболевания, исходя из приведенного анализа литературных данных.

Ключевые слова: коммуникативная функция речи, высшая нервная деятельность, когнитивные нарушения, острое нарушение мозгового кровообращения, комплексный подход.

Как правило, при ОНМК возникает целый комплекс когнитивных нарушений, характеризующийся нарушениями речи, снижением объема кратковременной и долговременной памяти, неадекватным социальным поведением, поражением когнитивных процессов, утратой способности логически мыслить и решать проблемы [9]. Имеются литературные данные $[2,18]$, согласно которым подобные каскадные нарушения обусловлены широким спектром триггерных аспектов: ухудшением активности практически всех важнейших нейротрансмиттерных систем (дофаминергической, серотонинергической, ГАМКергической), увеличением индукции провоспалительных цитокинов и развитием нейровоспалительной реакции, увеличением уровня свободных радикалов и появлением органических поражений головного мозга. При этом возникают и поведенческие нарушения: асоциальность, стереотипность поведения, депрессивные 
расстройства и нарушения речи.

В настоящее время усилия многих научных центров направлены на изучение этиопатогенетических механизмов, возникающих при ОНМК, а также на поиск способов коррекции и возвращению пациентов к прежнему уровню функциональной активности. При этом, учитывая гетерогенность и сложность возникающих нарушений, адекватным подходом для решения проблемы представляется создание комплексного индивидуального подхода к лечению и коррекции этой аномалии.

Именно поэтому большое значение имеет индивидуально-личностный подход к состоянию каждого пациента: лечащий специалист должен владеть информацией о его профессиональных интересах, значимых событиях личной жизни, преморбидных увлечениях и типе нервной системы. Особое внимание следует уделить изучению сопутствующих заболеваний при их наличии, в особенности тех, что накладывают серьёзные ограничения на образ жизни человека. К таким нарушениям можно отнести ряд системных патологий: сахарный диабет, заболевания сердечно-сосудистой системы, психические расстройства и зависимости. Для этого важно исследовать глубину повреждений ВПФ всеми доступными методами - МРТ, КТ, опрос больного и изучение состояния его когнитивных функций $[13,14]$.

Как правило, вместе с терапией лекарственными препаратами лечение должно проводиться и психосоциальными методами: для коррекции нейропсихологических нарушений и контроля общего состояния больного важно участие психолога, невропатолога, психиатра, логопеда и окружения пациента. Для составления качественной и количественной оценки общего состояния когнитивных процессов пациента, а также возможных прогнозов о дальнейшем лечении специалист должен применять широкий спектр тестов и методик.

Так для первичной экспресс-оценки когнитивных функций пациента целесообразно применение опросника MMSE (Mini Mental state Examination), шкалы деменции Матиса, батареи тестов для оценки лобных дисфункций, FAB (Frontal Assessment Bullory) и шкалы глобального клинического снижения (Global Deterioration Scale - GDS) $[4,15,19]$. Во избежание переутомления пациента и искажения результатов необходимо проводить диагностику в кратчайшие временные промежутки: не более 30 мин. в день [11].

При построении программы по восстановлению и корректировке ВПФ необходимо придерживаться ряда базовых принципов: постепенное усложнение задач, положительное подкрепление, вне зависимости от результата, а также подкрепление мотивации и коррекции функций, как при индивидуальной, так и при групповой форме работы. Кроме того, важной особенностью ОНМК является длительность процесса реконвалесценции когнитивных функций, потому специалист должен суметь донести до пациента и его окружения необходимость ежедневного лечения. При этом критериями эффективности программы будут являться не только восстановление когнитивных функций, но и субъективные положительные ощущения пациента в случае успеха [5].

Большую роль при работе с пациентом при любом типе нарушений ВПФ является установление личного контакта и доверия. Как правило, для установки доверительных отношений специалисту необходимо не только тонко чувствовать и понимать больного, но и провести серьёзную подготовительную работу - важно знать, кем пациент работает, сложности и особенности его деятельности, его интересы, увлечения, семейное положение и многое другое. В работе этот аспект поможет решить сразу два важных момента: таким образом, врач и пациент смогут говорить на одном языке и лучше понимать друг друга, а также существенно упрощается восстановление когнитивных функций, связанных с ранее известными пациенту моментами. Например, математик, в отличие от писателя, гораздо проще заново освоит примеры устного счёта.

Кроме того, в случае отсутствия видимого прогресса на определённом этапе кредит доверия к лечащему специалисту поможет сохранить контакт с больным. Именно поэтому начальный этап всех занятий должен быть предельно простым и не вызывать у пациента сложностей при освоении, что повышает энтузиазм и положительные эмоции пациента. На начальных этапах рекомендуется сохранять образцы почерка и голоса пациента для последующего сравнения. Этот аспект позволит наглядно продемонстрировать пациенту его прогресс и подстегнуть уверенность в своих силах.

Следует понимать, что восстановление ВПФ представляет собой сложный процесс, зависящий от многих факторов: локализации повреждения, обширности нарушения и длительности негативного воздействия, от личности пациента, его возраста, и даже того, знает ли он другие языки и какой рукой он ест. При этом, согласно литературным данным, существует прямая зависимость между ранним началом лечения и благоприятным и полным восстановлением ВПФ.

В качестве иллюстрации приведем клинический случай. Больной, мужчина 57 лет, страдающий повышением артериального давления до 240/110 мм, регулярно не обследовался. Поступил в стационар с диагнозом острое нарушение мозгового кровообращения в бассейне левой задней мозговой артерии. При первичном обследовании было установлено: пациент охотно вступает в контакт, ориентирован в месте, времени, собственной 
личности. Критика к своему состоянию достаточная, к обследованию мотивирован, допускаемые ошибки стремится исправить, принимает помощь специалиста. У больного выявляются нейродинамические нарушения: инертность, истощаемость, замедление темпа деятельности, увеличение латентного периода во всех видах деятельности. Произвольная регуляция деятельности не нарушена. Уровень психической активности в процессе обследования средний, отмечаются колебания произвольного внимания и работоспособности, необходим период осмысления задания. Поведение пациента в ситуации обследования адекватное. Больной правша, семейное левшество отрицает. Пациенту доступно понимание простых инструкций, сложных - затруднено. На восприятие речи оказывает влияние дефицитарность в сфере произвольного внимания, инертность, недостаточность в сфере переключения слухового внимания. Объем слухо-речевой памяти существенно ограничен. Спонтанная речь бедная, представлена отдельными частотными словами, выраженный лексический дефицит снижает возможности коммуникативной функции речи. Высказывание не всегда носит ситуативный характер, каких-либо произносительных трудностей не обнаруживается. Темп речи нормальный, интонация адекватна содержанию высказывания. Общие коммуникативные возможности нарушены. Диалогическая речь возможна, но ответы не всегда достаточно информативны. Речевые автоматизмы у больного полностью сохранны. Повторная речь в целом сохранена. У больного имеются выраженные трудности называния, преимущественно предметов, обусловленные недостаточностью в сфере зрительных представлений. У больного выявляются выраженные нарушения в сфере зрительного предметного гнозиса, а также буквенная агнозия.

В результате нейропсихологического обследования были выявлены амнестическая афазия, буквенная и предметная агнозия, нарушения нейродинамического компонента психической деятельности.

В процессе определения курса лечения и его осуществления принимал участие широкий спектр специалистов: терапевт, врач ЛФК, психотерапевт, афазиолог, психолог и младший медицинский персонал. Курс лечения, помимо соответствующей лекарственной терапии (пирацетам 1200мг/кг, актовегин 80 мг/кг, глицин 100мг/ кг) составил проведение ежедневных индивидуальных занятий на протяжении 8 недель по 60 минут в день.

Для восстановления оптических образов было использовано несколько методик: методики применения объёмных букв и моторного двигательного образа буквы (изображение буквы в воздухе) в сочетании с методом проговаривания, а также слуховой метод актуализации образа буквы. При использовании этих подходов отмечалось улучшение речи в виде уменьшения латентного периода при назывании предметов.

Большая часть работы была уделена закреплению оптического образа рукописной и печатной буквы. При этом сами буквы были рассмотрены не изолированно, а внутри слова, что требовало от пациента детального анализа при чтении и письме. В этом случае были применены методики вербального анализа при сравнении оптически сходных букв. Большая роль при коррекции этих нарушений уделялась написанию отдельных букв и простых по слоговой структуре слов с опорой на двигательный образ, например, написание букв в воздухе.

После его прохождения у пациента отмечалась положительная динамика - восстановление оптического образа различных бытовых предметов, выраженное в увеличении частоты использования названий бытовых предметов, уменьшение степени выраженности амнестической афазии, заключавшееся во введении в речь различных смысловых конструкций, а также заметное улучшение чтения и понимания прочитанного (от побуквенного прочтения слов был заметен переход к пословному варианту).

Существенно важную роль в восстановлении сыграло применение индивидуального подхода и использование "триггерных» материалов: так как пациент филолог, то специалистом был применён ряд заведомо ложных утверждений основных правил, терминов и фамилий известных филологов, что работало как усилитель внимания, побуждая пациента к исправлению, и как своеобразная стимуляция интереса в случае диалоговой формы работы.

Таким образом, раннее своевременное проведение комплексного восстановительного обучения при соблюдении ряда условий создаёт предпосылки для полного восстановления когнитивных функций, даже в случае вышеуказанного многоаспектного и сложного повреждения: амнестической афазии, буквенной агнозии и алексии.

В соответствии с имеющейся у пациента симптоматикой когнитивных нарушений была составлена программа комплексных мероприятий:

Проведение индивидуальных занятий 3 раза в неделю по 60 минут. Главный акцент занятий был сделан на индивидуально-психологический подход в лечении, который заключался в использовании базовых основ хорошо знакомой пациенту дисциплины - филологии. Например, затрагивались вопросы происхождения и изменения языков различных групп, принадлежность слов к тому или иному языку, особенности их образования и видоизменения. Кроме того, при работе большое внимание уделялось ряду конкретных вопросов и тер- 
минов: так как по своей профессии пациент был филологом-лингвистом, специализирующимся на исследовании языков англо-фризской группы, поэтому специалист на занятиях включал в работу особенности адаптации при распространении этих языков, в частности явление «пиджинов».

На более поздних этапах работы обсуждались основные работы известных филологов - Д.Р.Д. Толкиена, Р.Д. Эльяшевича, Г.М. Маклюэна. Специалист интересовался мнением больного, его позицией, а также основными сложностями при работе с новым материалом.

Кроме того, для реконвалесценции когнитивных функций был сделан акцент на арт-терапию для восстановления идеомоторного праксиса, усиления концентрации и скорости ориентирования во времени и пространстве. Кроме того, именно этот этап занятий является первым звеном в социализации пациента, а также формировании позитивной жизненной перспективы и корректировкой его речевых навыков. В начале каждого занятия были проведены упражнения, направленные на усиление концентрации внимания (оптическая и пальцевая гимнастика, музыко- и арт-терапия).

Для дальнейшего сохранения достигнутого прогресса была рекомендована работа с дидактическими тренажерами в соответствии с нейропсихологическими данными пациента. Суть тренировки заключается в восстановлении навыков самообслуживания и работе на тренажерах разной направленности:

- восстановление конструктивного праксиса, предметного гнозиса - складывание мозаики, конструктора;

- восстановление счётных навыков, логического мышления и укрепления внимания через игры лото, домино;

- восстановление зрительной памяти - сложение кубиков, архитектурное лото;

- восстановление бытовых навыков - работа с домашней утварью, столовыми приборами, деталя- ми одежды [10, 16, 17].

Несмотря на то, что психокоррекционная работа в стационарных условиях достаточно эффективна, пациенту необходимо обеспечить оттачивание его социальных навыков, что предполагает его общение с семьёй и окружением. Именно поэтому специалист должен доступно объяснить необходимость продолжения дальнейшего курса лечения в домашних условиях [3]. При этом важной деталью в работе с людьми, опекающими больного, будет являться аспект предоставления пациенту постепенно увеличивающейся свободы и приятие роли не надзирателя, а советника. Так, рекомендуется стимулировать пациента по мере его сил на самообслуживание, ведение с ним ежедневных бесед и предоставление ему выбора в принятии простых решений [7].

В результате становится очевидна значимость комплексного подхода при лечении симптомов и механизмов ОНМК и коррекции специфики их проявления. Очевидно, что только благодаря тщательному и педантичному подбору видов и способов активности для каждого конкретного пациента с учётом клинической картины его поражений, можно достичь восстановления прежнего уровня когнитивных функций. При этом, рассматривая синдром ОНМК как комплексную аномалию, вносящую целый каскад изменений в структуру личности, можно добиться возвращения к прежней жизни пациента и нивелированию негативной симптоматики [12].

Поскольку ВПФ человека представляют собой совокупность результатов взаимодействия факторов ментального и физического здоровья, его окружения и общего благополучия, то на их состояние способен оказывать влияние широкий спектр факторов. Принимая во внимание сложность и гетерогенность психических процессов, необходимо понимать, что при их коррекции следует применять комплексный и системный подход к разработке программы восстановительного обучения, что позволит достичь нужного результата в кратчайшие сроки.

\section{ЛИТЕРАТУРА}

1. Абдулаева Н.Н. Вязикова Н.Ф., Шмырина К.В. Особенности эпилепсии у лиц, перенёсших острое нарушение мозгового крово0бращения // Dobrokhotov readings. 2017. № 31. C. 11-18.

2. Антонова К.В., Танашян М.М., Романцова Т.И., Максимова М.Ю. Острые нарушения мозгового кровообращения: клинические течения и прогноз у больных сахарным диабетом 2 типа// Ожирение и метаболизм. 2016. № 2. С. 13.

3. Вахнина Н.В., Парфенов В.А., Никитина Л.Ю. Когнитивные нарушения при инсульте и их лечение мемантином // Клиническая геронтология. 2005. № 11(8). C. 49-52.

4. Голубятникова В.И., Кузнецова А.А. Основные подходы к диагностике нарушений речи и других высших психических функций в структуре помощи больным с острым нарушением мозгового кровообращения // Коллекция гуманитарных исследований. 2017. № 1. С. 4.

5. Джурабекова А.Т., Шмырина К.В., Рашидова С.И. Острые нарушения мозгового кровообращения у детей // Актуальные проблемы медицинской науки и образования. 2019. № 4. С. 238-240. 
6. Ермакова Н.Г. Психотерапевтическая среда при реабилитации больных с последствиями инсульта-одна из форм терапии средой // Вестник Южно-Уральского государственного университета. Серия: Психология, 2016. № 9(2). С.8.

7. Ермакова Н.Г. Психологическая коррекция нарушения высших психических функций больных с последствиями инсульта в нейрореабилитации (теоретико-методологические аспекты) // Вестник Санкт-Петербургского университета. Социология, 2008. № 2. С.345-353.

8. Ковальчук В.В., Гусев А.О., Минуллин Т.И., Нестерин К.В. Реабилитация пациентов после инсульта. Критерий эффективности и факторы успеха: роль физической и медикаментозной терапии// Эффективная фармакотерапия. 2017. № 19. С.62-73.

9. Мачинский П.А., Плотникова Н.А., Ульянкин В.Е., Кемайкин С.П., Рыбаков А.Г. Сравнительная характеристика показателей смертности и летальности от ишемического и геморрагического инсультов в России // Известия высших учебных заведений. Поволжский регион. Медицинские науки, 2019. № 3(51). C. 101-118.

10. Никитин М.В., Шонгина Н.Н. Чукина И.М. Восстановление речи и других высших психических функций у пациентов, перенесших острое нарушение мозгового кровообращения, на стадии реабилитации в условиях санаторного комплекса // Colloquium journal. 2019. № 5. C. 29.

11. Шмырина К.В., Вязикова Н.Ф., Абдулаева Н.Н., Исламова Д.С. Роль среднего медицинского персонала в реабилитации пациентов с последствиями перенесённого острого нарушения мозгового кровообращения. // Здоровье, демография, экология финно-угорских народов. 2019. № 4. С. 21 -24.

12. Custodio N., Bererra-Becerra Y., Alva-Diaz C., Montesion R., Lira D., Herrera-Perez E., Castro-Suarez S. Validation and diagnostic accuracy of the global deterioration scale (GDS) to establish severity of dementia in a population of Lima // CES Medicina, 2017. 31(1). P. 14-26.

13. Honda M., Ichibayashi R., Suzuki G., Toyoda Y., Watanab M., Serizawa H., Seiki Y. Мозговое кровообращение у больных черепно-м0зговой травмой с острой субдуральной гематомой: ксеновая КТ и перфузионная КТ-исследование // Скорая нейрохирургическая помощь. 2018. № 23(1). С. 1-9.

14. Martin A., Domercq M., Matute C. Inflammation in stroke: the role of cholinergic, purinergic and glutamatergic signaling // Therapeutic advanages in neurological disorders, 2018. № 11. C. 67.

15. Mitchell A.J. The mini-Mental State Examination (MMSE): update on its diagnostic accuracy and clinical utility for cognitive disorders. In Cognitive screening instruments, Springer, Cham, 2017. P. 37-48.

16. Nadareishvili Z., Luby M., Leigh R., Shah J., Lynch J.K., Hsia A.W., Latour L. L. An MRI hypertense acute reperfusion marker is related to elevated peripheral monocyte count in acute ischemic stroke // Journal of Neuroimaging. 2018. 28(1). P. 57-60.

17. Sten K., Just J., Blauenfeld R.A., Drasbek K.R. Extracellular Vesicle in acute Stroke Diagnostics // Biomedicines. 2020. 8(8). P. 248.

18. Tarvolen-Scroder S., Hurne S., Laimi K. The World Health Organization disability assessment schedule (WHODAS 2.0) and the WHO minimal generic set of functioning and health versus conventional instruments in subacute stroke // Journal of rehabilitation medicine. 2019. № 51(9). P. 675-682.

19. Zhao L., Xiong Q., Staty C.M., Mahgoub 0.K., Ye Y., Gu L., Zhu S. Bidirectional gut-brain-microbiota axis as a potential link between inflammatory bowel disease and ischemic stroke // Journal of Neuroimaging, 2018. № 28(1). P. 57-60.

20. Zhu Y.,Zhao S., Fan Z., Li Z., He F., Lin C., Zhang B. Evaluation of the Mini-Mental State Examination and the Montreal Cognitive Assessment for Predicting Poststroke Cognitive Impairments During the Acute Phase in Chinese Minor Stroke Patients. Frontiers in aging neuroscience. 2020. № 12, P. 236.

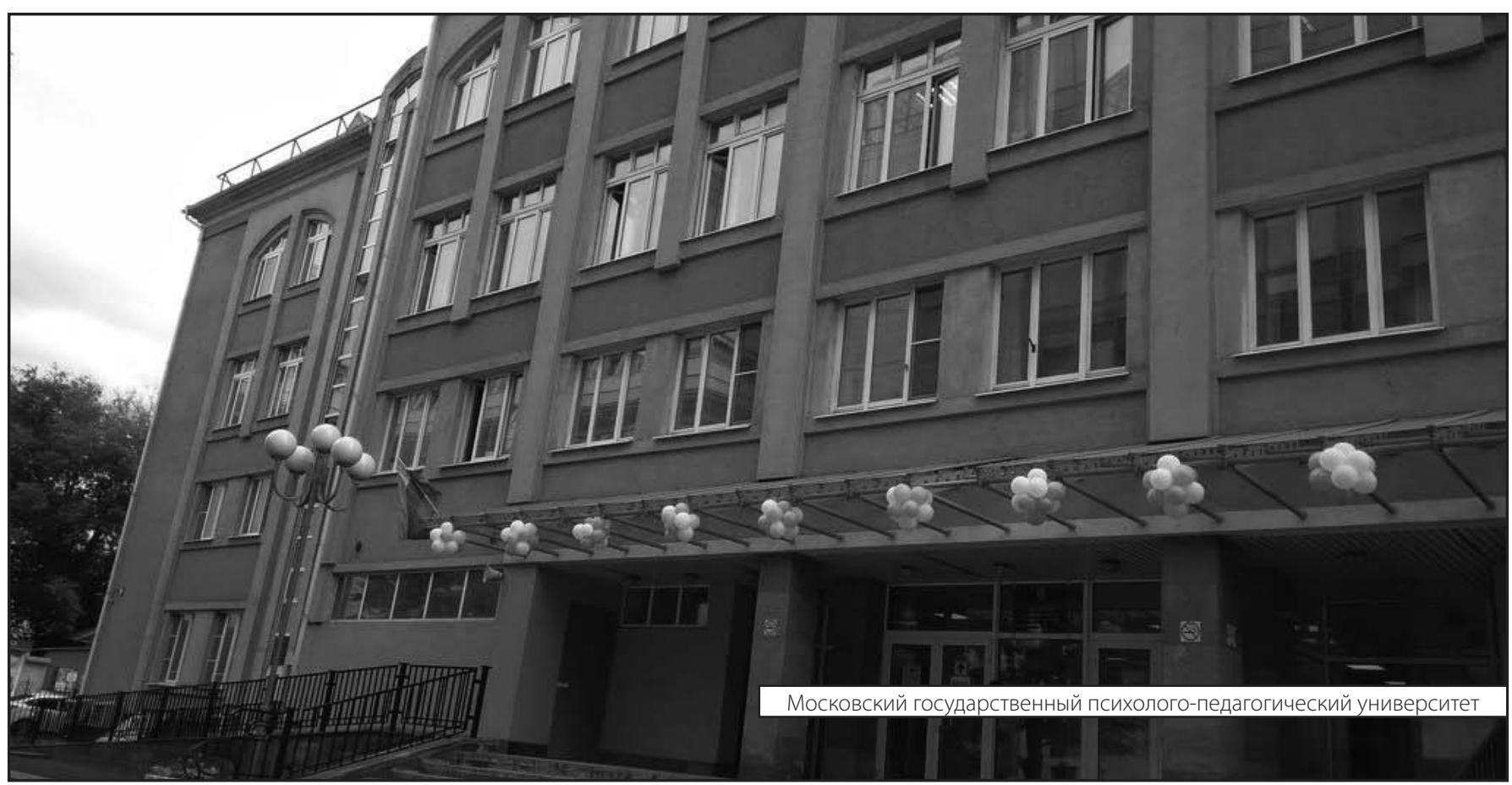

